\title{
UGT1A1*28 Polymorphism in Advanced Colorectal Cancer: The Story Is Not Yet Ended
}

\author{
Ahmed El Bastawisy ${ }^{1 *}$, Abeer Bahnasy ${ }^{2}$, Amany El-Zeiny ${ }^{3}$, Samar Farid ${ }^{4}$ \\ ${ }^{1}$ Medical Oncology, National Cancer Institute, Cairo University, Cairo, Egypt; ${ }^{2}$ Pathology, National Cancer Institute, Cairo Univer- \\ sity, Cairo, Egypt; ${ }^{3}$ Clinical Pharmacy, National Cancer Institute, Cairo University, Cairo, Egypt, ${ }^{4}$ Clinical Pharmacy, Faculty of \\ Pharmacy, Cairo University, Cairo, Egypt. \\ Email: ${ }^{*}$ a s basta@hotmail.com
}

Received November $18^{\text {th }}, 2013$; revised December $15^{\text {th }}, 2013$; accepted December $23^{\text {rd }}, 2013$

Copyright (C) 2014 Ahmed El Bastawisy et al. This is an open access article distributed under the Creative Commons Attribution License, which permits unrestricted use, distribution, and reproduction in any medium, provided the original work is properly cited. In accordance of the Creative Commons Attribution License all Copyrights (C) 2014 are reserved for SCIRP and the owner of the intellectual property Ahmed El Bastawisy et al. All Copyright (C) 2014 are guarded by law and by SCIRP as a guardian.

\section{ABSTRACT}

Background: UGT1A1*28 polymorphism is associated with neutropenia and diarrhea in previous reports, while this study tried to investigate correlation with other toxicities like vomiting. Patients and Methods: This is a prospective case control study including all eligible cases of advanced colorectal cancer. The genotypes of UGT1A1*28 was assessed in the peripheral blood and/or in tissues by PCR. Patients were divided into two groups, Group 1: patients with no mutation, Group 2: patients with homo or hetero mutation. All patients received standard IFL regimen. Primary objectives were: 1) comparison between the 2 groups as regard vomiting, 2) assessment of the incidence of UGT1A1*28 polymorphism. Secondary objectives were: comparison between the 2 groups as regard: neutropenia, diarrhea, treatment delay, progressive diseases (PD), progression free survival (PFS) and overall survival (OS). Results: 46 cases of advanced colorectal cancer present to National Cancer Institute, Cairo University, aged between 19 and 71 years with a median age of 45 years were included and followed up during the period from September 2010 to January 2013 with a median follow-up of 9 months. UGT1A1* 28 polymorphism was present in 20 patients (43\%), of whom $15 \%$ are homozygous. Grade (II-IV) vomiting was found in $8.3 \%$ of Group 1 versus $52.5 \%$ of Group $2(P=0.01)$. Grade (II-IV) neutropenia was found in $20.8 \%$ of Group 1 versus $64.7 \%$ of Group $2(P=0.03)$. Grade (II-IV) diarrhea was found in $37.5 \%$ of patients of Group 1 and $27.5 \%$ of patients with Group 2. $(P=0.75)$. Treatment delay occurred in $29.16 \%$ of Group 1 versus $72.4 \%$ of Group $2(P=0.02)$. $25 \%$ of Group 1 showed PD versus $25 \%$ of Group $2(P=0.8)$. 1-year PFS was $19 \%$ in Group 1 versus $23 \%$ in Group $2(P=0.8)$ while there was a trend towards better OS in Group 1 $(47 \%$ versus $35 \%)(P=0.07)$. Conclusions: UGT1A1*28 polymorphism is present frequently $(43 \%)$ in a Caucasian population and is associated with more vomiting, neutropenia and treatment delay.

\section{KEYWORDS}

UGT1A1*28 Polymorphism; Vomiting; Colorectal Cancer

\section{Introduction}

Colorectal cancer is the fourth most frequently diagnosed cancer and the second leading cause of cancer-related death in the United States [1].

Because Irinotecan-based chemotherapy is a standard treatment in the management of metastatic CRC, interpatient variation of the enzymes involving its metabolism has been investigated extensively. Irinotecan (CPT-11) is

"Corresponding author. a water-soluble analogue of 20(S)-camptothecin (CPT) and is an inactive prodrug. Its major metabolite, SN-38, is a potent active topoisomerase I-inhibitor and is known to be toxic [2]. One limitation of CPT-11 is the unpredictable and occasionally fatal gastrointestinal and hematologic toxicity, which varies greatly among individuals [3]. Predictive markers of CPT-11 toxicity, may thus be deduced from the CPT-11 metabolic pathway.

$\mathrm{SN}-38$ undergoes glucuronide conjugation to the pharmacologically inactive $\mathrm{SN}-38$ glucuronide by the hepatic 
uridine diphosphate glucuronosyltransferase 1A1 (UGT 1A1) enzyme. The glucuronidation of SN-38 may be protective against irinotecan-induced gastrointestinal toxicity [4]. This protection may be genetically determined, as UGT1A1 enzyme is known to exist in polymorphic states.

The specific polymorphism (UGT1A1*28) is the presence of a (TA)7TAA sequence in the promoter region, instead of (TA)6TAA. Studies with human liver microsomes, have demonstrated an association between this polymorphism and SN-38 glucuronidation, with significantly lower SN-38 glucuronidation rates in liver samples heterozygous or homozygous for the (TA)7TAA polymorphism [5]. Thus, knowledge of the UGT1A1 polymorphism status could help guide the selection of appropriate starting dosages, reducing the risk of severe toxicity and improving the chances that therapy could be maintained.

Roughly $10 \%$ of the US population is homozygous for UGT1A $1 * 28$. The frequency of the UGT1A $1 * 28$ allele varies among ethnicities, being highest in those of African (43\%) or European (39\%) descent and lowest in those of Asian (16\%) descent [6].

Among diplotypes of UGT1A genes, patients with the haplotypes harboring UGT1A $1 * 6$ or $* 28$ had significantly reduced area under concentration curve ratios, with the effects of UGT1A $1 * 6$ or $* 28$ being of a similar scale. In multivariate analysis, the homozygotes and double heterozygotes of $* 6$ and $* 28(* 6 / * 6$, $* 28 / * 28$ and $* 6$ / *28) were significantly associated with severe neutropenia in patients who received irinotecan monotherapy [7].

\section{Aim of Work}

Primary objectives were:

1) Comparison between patients with wild and mutant UGT1A $1 * 28$ as regard vomiting.

2) Assessment of the incidence of UGT1A1*28 polymorphism.

Secondary objectives were:

Comparison between the 2 groups as regard: neutropenia, diarrhea, treatment delay, progressive diseases (PD), progression free survival (PFS) and overall survival (OS).

\section{Patients and Methods}

This is a prospective case control study including all eligible cases of advanced colorectal cancer. The genotypes of UGT1A1*28 was assessed in the peripheral blood and/or in tissues by PCR. Patients were divided into two groups, Group 1: patients with no mutation, Group 2: patients with homo or hetero mutation.

The study was conducted according to the Declaration of Helsinki and the guidelines for Good Clinical Practice. The local ethics committees approved the protocol, and informed consent was obtained from all patients before study entry.

\subsection{Inclusion Criteria}

Patient must have a histologically confirmed diagnosis of colon cancer. Stage III \& Stage IV colon cancer. Patient is at least 18 years of age. Patient has a performance status of $($ ECOG Scale) $\leq 2$. Patient has adequate bone marrow function, (WBC count $\geq 3.0 \times 10^{9} / \mathrm{L}$, ANC $\geq 1.5$ $\times 10^{9} / \mathrm{L}$ platelet count $\geq 100 \times 10^{9} / \mathrm{L}$, hemoglobin level $\geq 9 \mathrm{~g} / \mathrm{L}$ ). Patient has adequate liver function; serum bilirubin $<1.5 \times$ ULN, ALT and AST levels $<$ three times normal values; ALT and AST levels $<$ five times normal limits allowed in patients with known liver metastases. Patient has adequate Kidney function; plasma creatinine level $<1.5$ times normal value. Patients should have compliance, mental state and geographic proximity that allow adequate follow up and they have to provide written informed consent before any study-specific procedure.

\subsection{Exclusion Criteria}

Patient is pregnant or breastfeeding. Patients with a "currently active" second malignancy. Patient who is currently involved in another clinical trial.

\subsection{Treatment Plan}

All patients received standard IFL (Irinotecan, 5FU, Leucovorin) regimen: Irinotecan $125 \mathrm{mg} / \mathrm{m}^{2}$ weekly, Leucovorin $20 \mathrm{mg} / \mathrm{m}^{2}$ weekly and $5 \mathrm{FU} 425 \mathrm{mg} / \mathrm{m}^{2}$ weekly 4 weeks on 2 weeks off up to 6 months in responding patients.

\subsubsection{Study Assessment}

Pretreatment assessment included complete medical history and physical examination.

Further assessment conducted within 7 days before treatment included vital signs, performance status (ECOG), complete blood count with differential and full biochemical panel, including liver and renal function tests were performed and repeated before each treatment course. CEA and CA19.9 were done at base line and then every 6 weeks. Radiological evaluation including computerized tomography (CT) scan of the chest, Abdomen \& pelvis. Additional radiological imaging such as bone scan... were done if indicated. Imaging was repeated every 6 weeks.

Evaluation was done according to RECIST [8].

\section{Post treatment evaluation included:}

Medical history and physical examination every 3 weeks. CBC and chemistry every 3 weeks. CT chest, abdomen and pelvis every 6 weeks. CEA and CA19.9 every 6 weeks. Other investigations were done if indicated. 


\subsubsection{Toxicity}

Toxicity evaluation was done according to the NCI Common Terminology Criteria for Adverse Events v4.0 (CTCAE) [9].

\subsubsection{Statistical Methods}

SPSS package (version 17.0) was used for data analysis. Mean and standard deviation were reported to describe quantitative data. The Chi-square and Fischer exact tests were used to evaluate the differences in the distribution of the variables. The Kaplan-Meier method was used to estimate the overall and progression free survival and the Log rank test to evaluate differences in survival among groups. $P$ value of $\leq 0.05$ was considered statistically significant.

\subsubsection{UGT1A1 Assessment}

UGT1A1*28 polymorphism is characterized by the presence of an additional TA repeat in the TATA sequence of the UGT1A1 promoter ((TA)7TAA, instead of (TA)6TAA). In the current study, UGT1A1*28 polymorphism was assessed in the blood and/or tissues by PCR as previously described by Iyer et al., 2002 [10] and Akiyama et al., 2008 [11].

1) Extraction of DNA from blood Mononuclear cells (MNCs)

Blood samples $(7 \mathrm{ml})$ were collected from each patient on day 1 of the cycle (before starting chemotherapy). Density gradient separation of the MNCs from the collected blood was done using FicollHypaque Solution. DNA was extracted from the MNCs by phenol: chloroform: isoamyl alcohol after proteinase $\mathrm{K}$ digestion according to standard protocols. The extracted DNA was used to detect UGT1A1*28 polymorphism by PCR.

2) DNA Extraction from formalin fixed paraffin embedded tissues (FFPET)

For each tumor sample included in the study, five micron thick sections (5 sections) were obtained in a sterile, eppindorff, plastic tube. DNA was extracted from the homogenized FFPET sections by phenol: chloroform: isoamyl alcohol after proteinase $\mathrm{K}$ digestion according to standard protocols. Assessment of the concentration and purity of the extracted DNA was done using the spectrophotometer followed by visualization of an ethidium bromide-stained gel.

3) PCR amplification and genotyping of UGT1A1*28

The isolated DNA was amplified by polymerase chain reaction (PCR) using the primer sequences and conditions of Iyer et al., 1999 [5].

Primers:

5'-GTC ACG TGA CAC AGT CAA AC-3'

5'-TTT GCT CCT GCC AGA GGT T-3'

PCR conditions
Initial denaturation step at $95^{\circ} \mathrm{C}$ for 5 minutes, 30 cycles of: $95^{\circ} \mathrm{C}$ for 30 seconds, $58^{\circ} \mathrm{C}$ for 40 seconds, $72^{\circ} \mathrm{C}$ for 40 seconds and final extension at $72^{\circ} \mathrm{C}$ for 5 minutes.

Genotypes were assigned as follows:

6/6: homozygous for (TA)6TAA.

6/7: heterozygous for each (TA)6TAA/(TA)7TAA.

7/7: homozygous for (TA)7TAA.

5/8: heterozygous for each (TA)5TAA/(TA)8TAA.

\section{Results}

46 of advanced colorectal cancer cases presenting to National Cancer Institute, Cairo University, aged between 19 and 71 years with a median age of 45 years were included and followed up during the period from September 2010 to January 2013 with a median follow up of 9 months.

\subsection{Patients' Characteristics}

Table 1 summarizes patients' characteristics with regard to age, sex, stage, pathology, side, and smoking history.

\subsection{Incidence of UGT1A1 Polymorphism}

UGT1A1*28 polymorphism were present in 20 patients (43\%), homozygous in 7 patients (15\%) and heterozygous in 13 patients (28\%) (Figure 1).

Genotypes were:

6/6 in 26 patients (57\%).

$6 / 7$ in 9 patients (20\%).

$7 / 7$ in 7 patients $(15 \%)$.

Table 1. Patients' characteristics.

\begin{tabular}{|c|c|c|c|c|}
\hline & \multirow{2}{*}{ Characteristic } & \multicolumn{2}{|c|}{ No. of patients (\%) } & \multirow{2}{*}{ P-value } \\
\hline & & Wild & Mutant & \\
\hline & All patients & $26(100)$ & $20(100)$ & \\
\hline & Mean age & 45 years & 44 years & \\
\hline \multirow{2}{*}{ Sex: } & Male & $14(54)$ & $12(60)$ & 0.4 \\
\hline & Female & $12(46)$ & $8(40)$ & 0.4 \\
\hline \multirow{3}{*}{ Stage: } & III & $10(38)$ & $6(30)$ & 0.39 \\
\hline & IV & $16(62)$ & $14(70)$ & 0.39 \\
\hline & Adenocarcinoma GII & $17(66)$ & $12(60)$ & 0.4 \\
\hline \multirow[b]{2}{*}{ Pathology: } & Adenocarcinoma GIII & $4(17)$ & $2(10)$ & 0.4 \\
\hline & $\begin{array}{c}\text { Mucinous } \\
\text { Adenocarcinoma GII }\end{array}$ & $4(17)$ & $5(25)$ & 0.3 \\
\hline \multirow{4}{*}{ Side: } & Undifferentiated Carcinoma & - & $1(5)$ & 0.4 \\
\hline & Left & $7(27)$ & $6(30)$ & 0.5 \\
\hline & Right & $19(73)$ & $14(70)$ & 0.5 \\
\hline & Smoking history & $4(15)$ & $3(15)$ & 0.4 \\
\hline
\end{tabular}


$5 / 8$ in 4 patients (8\%).

\subsection{Toxicity}

Vomiting: There was a statistically significant lower incidence of vomiting in patients with wild type UGT$1 \mathrm{~A} 1 * 28$ compared to patients with mutant type: Grade (II-IV) vomiting was found in $8.3 \%$ of Group 1 versus $52.5 \%$ of Group $2(\mathrm{P}=0.01)$ (Table 2$)$.

Neutropenia: There was a statistically significant lower incidence of neutropenia in patients with wild type UGT1A1*28 compared to patients with mutant type:

Grade (II-IV) neutropenia were found in $20.8 \%$ of Group 1 versus 64.7\% of Group $2(\mathrm{P}=0.03$ ) (Table 2).

Diarrhea: Grade (II-IV) diarrhea was found in $37.5 \%$ of patients of Group 1 and 27.5\% of patients with Group $2(\mathrm{P}=0.75)$ (Table 2).

\subsection{Treatment Delay}

There was a statistically significant lower incidence of treatment delay in patients with wild type UGT1A1*28 compared to patients with mutant type:

Treatment delay occurred in $29.16 \%$ of Group 1 versus $72.4 \%$ of Group $2(\mathrm{P}=0.02)$ (Table 2$)$.

Other toxicities included 1 case of thrombocytopenia grade III (in Group 1), 1 case of oral mucositis grade II

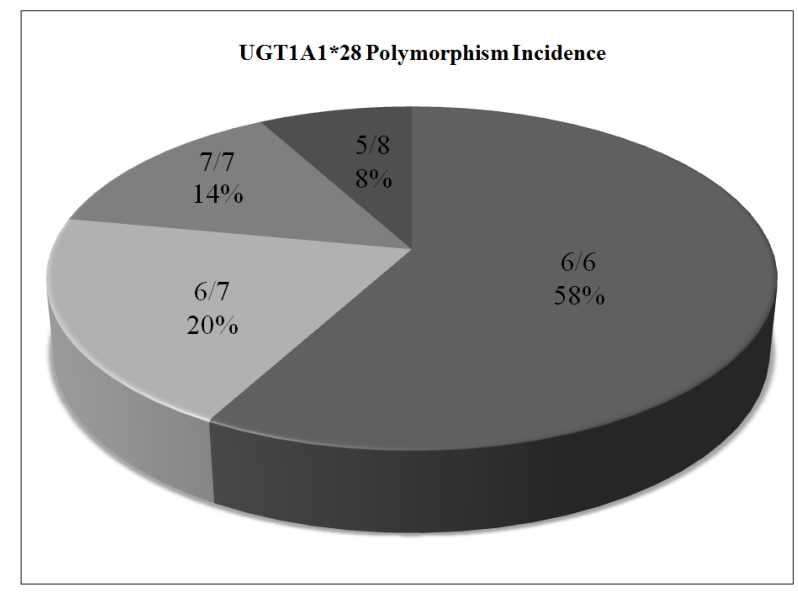

Figure 1. UGT1A1*28 polymorphism incidence.

Table 2. Correlation between UGT1A1*28 polymorphism and toxicities (Grades II, III, IV).

\begin{tabular}{cccc}
\hline Toxicity & Wild & Homo + Hetero & P value \\
\hline Diarrhea & $37.5 \%$ & $27.5 \%$ & 0.7523 \\
Neutropenia & $20.8 \%$ & $64.7 \%$ & $0.0325^{*}$ \\
Vomiting & $8.3 \%$ & $52.5 \%$ & $0.0109^{*}$ \\
Treatment delay & $29.16 \%$ & $72.4 \%$ & $0.0241^{*}$ \\
Dose reduction & $12.5 \%$ & $32 \%$ & 0.2502 \\
\hline
\end{tabular}

(in Group 1), 1 case of nausea grade II (in Group 2), 2 case of urinary tract infection (in Group 1), 2 cases of gastritis grade II (1 in each group) and 2 cases of abdominal pain grade II (in Group 2).

\subsection{Clinical Response}

There was no significant difference between the 2 groups as regard clinical response. 25\% of Group 1 showed PD versus $25 \%$ of Group $2(\mathrm{P}=0.8)$ (Table 3$)$.

\subsection{Survival}

Progression free survival (PFS): There was no significant difference between the two groups as regard PFS. 1 year PFS was 19\% in Group 1 versus 23\% in Group 2 (P $=0.8$ ) (Figure 2).

Overall survival (OS): There was a trend towards better OS in patients with wild type UGT1A1*28 compared to patients with mutant type: One year OS in Group 1 was $47 \%$ versus 35\% in Group $2(\mathrm{P}=0.07)$ (Figure 3 ).

\section{Discussion}

The main problem with Irinotecan, which is an important drug in the treatment of colon cancer, is its toxicity. Patients receiving Irinotecan may experience some kind of severe toxicity, mainly diarrhea and neutropenia, resulting either in dose reduction, treatment withdrawal or dy-

Table 3. Clinical response.

\begin{tabular}{ccc}
\hline Response & Wild & Hetero + Homo \\
\hline CR & $\mathbf{1 0 \%}$ & $\mathbf{1 5 \%}$ \\
PD & $\mathbf{2 5 \%}$ & $\mathbf{2 5 \%}$ \\
PR & $\mathbf{3 0 \%}$ & $\mathbf{3 5 \%}$ \\
SD & $35 \%$ & $\mathbf{2 5 \%}$ \\
\hline
\end{tabular}

$\mathrm{P}=0.8927$.

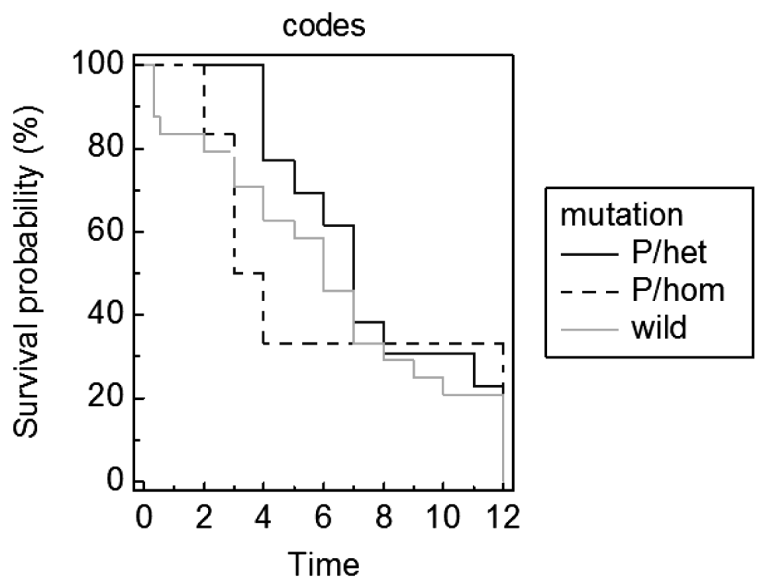

Figure 2. One year progression free survival. 


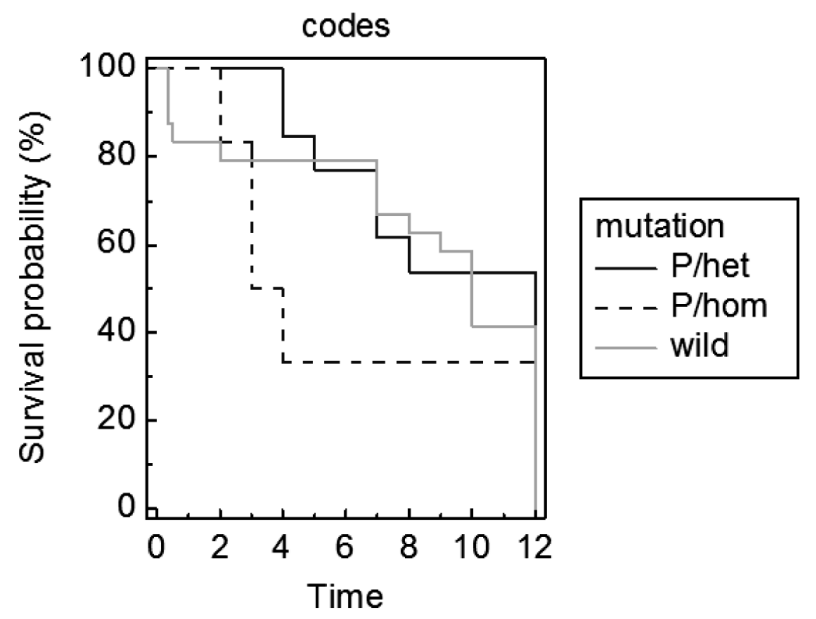

Figure 3. One year overall survival.

ing. UGT1A1*28 polymorphism is associated with neutropenia and diarrhea in previous reports, our study tried to investigate correlation with other toxicities like vomiting.

This is a prospective case control study including all eligible patients presenting to National Cancer Institute (NCI), Cairo University during the period from September 2010 to January 2013. Patients were divided into 2 groups according to the condition of UGT1A $1 * 28$ gene polymorphism to wild (6/6) and mutant genotype (6/7 and 7/7).

UGT1A1*28 polymorphism were present in $43 \%$ of patients, $15 \%$ homozygous (7/7) and 28\% heterozygous (6/7). In Christoph Schulz et al. (2009) study, 9.5\% of patients were homozygous and $49.5 \%$ were heterozygous for UGT1A1*28 [19]. In other studies, UGT1A1*28 polymorphism was present in a lower percentage such as $\mathrm{Y}$. Akiyama et al. (2008) study, in which there was $16.4 \%$ of Japanese patients having this polymorphism, and $10.5 \%$ in Xiaoqing Zhang et al. (2012) study (Han population), $15.3 \%$ in Zhang A et al. (2007) study (Dong population), $28.6 \%$ in Tang et al. (2005) study (Taiwanese population), $18.7 \%$ in Zhou et al. (2009) study (Singaporean population), 13\% in Saeki et al. (2006) study (Japanese population), $14 \%$ in Yea et al. (2008) study (Korean population) and $28.6 \%$ in Thomas et al. (2006) study (Caucasian population) [11-19]. It is noteworthy that ethnic differences do exist in the UGT1A1*28 polymorphism, that's why there is differences between different studies of different ethnicities.

To no surprise, the current study showed that, UGT$1 \mathrm{~A} 1 * 28$ polymorphism plays a significant role in the occurrence of hematological toxicity in patients treated with Irinotecan, especially neutropenia ( $20.8 \%$ vs. $64.7 \%$, $\mathrm{P}=0.03$ ), this finding is in concordance with previous studies which showed similar results such as Chun-Yu et al. (2007) (53.8\% vs 4.9\%; P < 0.01), Innocenti et al.
(2004) which stated that patients with the $7 / 7$ genotype had a 9.3-fold higher risk (95\% confidence interval, 2.4 36.4) of developing grade 4 neutropenia than patients with the 6/6 and 6/7 genotypes, L Iyer et al. (2001) in which there was a correlation between absolute neutrophil count (ANC) and UGT1A1 promoter genotypes in patients after irinotecan treatment with Significant trend in ANC nadirs $(6 / 6>6 / 7>7 / 7, \mathrm{z}=-2.05, \mathrm{P}=0.04$, non-parametric trend analysis), Kristine et al. (2006) with increased risk of grade 3 or 4 neutropenia ( $\mathrm{P}=$ 0.001 ) and Rouits et al. (2004) that confirmed statistically significant risk of neutropenia $(P=0.001)$ [10,19-23].

The current study showed no significant difference between the 2 study groups regarding risk of diarrhea (incidence $37.5 \%$ vs. $27.5 \%, \mathrm{P}=0.75$ ) which may be attributed to small sample size of the present study. Some previous studies shows no difference like Christoph Schulz et al. (2009) in which result $[(6 / 7,7 / 7)$ vs $(6 / 6)$ plymorphismwas $13.0 \%$ vs $6.2 \%, \mathrm{P}=0.08$ ] and Rouits et al. (2004) that found no significant statistical difference $(P=0.559)$. $(9,14)$ Other studies showed a statistically significant difference like: Chun-Yu et al. (2007) study which showed a statistically significant difference regarding diarrhea $(26.9 \%$ vs $5.9 \%$; $\mathrm{P}<0.01)$. Kristine et al. (2006) showed that there was an increased risk of grade 3 or 4 diarrhea $(\mathrm{P}=0.005)$. L. lyer et al. (2001) stated that patients with the (TA) 7TAA repeat in the promoter region of UGT1A1 may be expected to exhibit a higher incidence of diarrhea and neutropenia than those without the polymorphism [10,20,21].

The interesting finding in this study is that, although all patients received premedication antiemetic, patients with UGT1A1*28 polymorphism had significantly more risk for vomiting than patients with wild type $(52.5 \%$ in mutant type group versus $8.3 \%$ in the wild type group with $\mathrm{P}$ value of 0.01 .). To our knowledge this is the first study to primarily address correlation between UGT$1 \mathrm{~A} 1 * 28$ polymorphism and vomiting.

The current study showed no significant difference between the two groups regarding toxicity related dose adjustments $(12.5 \%$ versus $32 \%, \mathrm{P}=0.25)$ whereas there was a statistically significant more dose delay in the mutant group ( $29 \%$ versus $72 \%, \mathrm{P}=0.02)$. These results are to a large extent similar to those of previous studies: In Christoph Schulz et al. (2009) study, treatment delays incidence was; $25.1 \%$ vs. $19.3 \%$, [P $=0.24]$ in $[(6 / 7,7 / 7)]$ group vs. (6/6) group respectively and dose reductions incidence was $21.5 \%$ vs $27.2 \%$, [P $=0.07]$ in $[(6 / 7,7 / 7)$ group vs. (6/6) group respectively. Toffoli et al. (2006) showed that dose reduction occurred in $17.5 \%, 23.2 \%$, and $18.2 \%$ of TA6/TA6, TA7/TA6, and TA7/TA7 patients, respectively, with no significant association with genotypes. Chun-Yu et al. (2007) study states that requirement for irinotecan dose reduction was significantly 
greater in patients who had the genetic variant $(42.3 \%$ vs 12.7\%; P < 0.01) $[19,20,26]$.

Although the treatment delay was significantly greater in patients with the TA6/TA7 or TA7/TA7 genotypes when compared with wild type $(72.4 \%$ versus $29.16 \%$ respectively), $(\mathrm{P}=0.02)$ there was no significant difference between the two groups as regard clinical response, $\mathrm{P}$ value $=0.89$. This goes with most of previous published studies like Christoph Schulz et al. (2009) study, which stated that the overall response rate (OR) was similar between patients carrying the $(6 / 7,7 / 7)$ or the wild genotype (6/6) $(44.3 \%$ vs $43.2 \%, \mathrm{P}=0.75)$ and Chun-Yu et al. (2007) study which showed that the response rate to irinotecan-based chemotherapy were not affected with dose reduction that was required for the UGT1A $1 * 28$ polymorphism group $(42.3 \%$ vs $45.1 \%$; $\mathrm{P}=0.80)$. $(9,10)$ But some studies such as Glenn et al. (2009) reported that, the *28 heterozygotes had a nonsignificantly higher response rate (RR, 1.09; 95\% CI $0.83-1.43$ ), and *28 homozygotes had a significantly higher response rate (RR, 1.70; 95\% CI 1.24 - 2.33; P = < 0.001), and Toffoli et al. (2006) stated that, the UGT1A1 TA7/TA7 genotype seemed to be associated with increased clinical benefit and tumor response, as homozygous TA7/TA7 patients had a significantly reduced risk of PD or SD compared with the wild-type genotype (OR, 0.32; 95\% CI, 0.12 to 0.86 ) and considering clinical benefit, the homozygous TA7/TA7 patients had a significantly lower risk of experiencing progression (OR, 0.19; 95\% CI, 0.04 to 0.89) [24].

In our study, we did not find a statistically significant difference between the two groups as regard PFS and OS, (PFS: $19 \%$ in wild group vs. $23 \%$ in mutant group, $\mathrm{P}=$ 0.8 ), although there was a trend toward a statistical significant difference for OS at one year (wild: $47 \%$ vs. mutant: $35 \%, \mathrm{P}=0.07)$. It confirms the previous studies results. In Christoph Schulz et al. (2009) study neither time to progression [(TTP) 8.1 vs. 8.2 months, $\mathrm{P}=0.97$ ] nor overall survival [(OS) 21.2 vs. 18.9 months, $\mathrm{P}=0.73$ ] differed significantly in patients who carried the (6/6) or (6/7, 7/7) genotypes respectively. Chun-Yu et al. (2007) study stated that although the requirement for irinotecan dose reduction was significantly greater in patients who had this genetic variant, it did not significantly affect progression-free survival (10 months vs 11 months; $\mathrm{P}=$ 0.94 ) or overall survival (19 months vs 18 months; $\mathrm{P}=$ 0.84). The data from Toffoli et al. (2006) and Marcuello et al. (2004) studies reported that none of the differences were statistically significant. Findings from Toffoli et al. (2006) study were in the direction of improved survival for *28 homozygotes versus non wild genotype patients, whereas the Marcuello et al. (2004) study reported a survival advantage for the wild genotype individuals $[19,20$, $25,26]$.

\section{Conclusion}

The current study showed that UGT1A1*28 polymorphism is present frequently (43\%) in a Caucasian population and is a key determinant for predicting irinotecaninduced toxicities as vomiting, neutropenia and treatment delay for patients with advanced colorectal cancer with a trend toward improvement of survival. Further prospective studies including larger sample size are warranted for using this polymorphism to optimize irinotecan-based chemotherapy with special emphasis on other toxicities which may be related to this polymorphism.

\section{Acknowledgements}

This work was presented as poster at ESMO 15th World Congress on Gastrointestinal Cancer, 3-6 July 2013, Barcelona, Spain.

We thank Dr. Nelly Hassan, Professor of Medical Biostatistics, NCI, Cairo University for preparation of the statistics of this work.

\section{REFERENCES}

[1] R. Siegel, D. Naishadham and A. Jemal, "Cancer Statistics,” CA: A Cancer Journal for Clinicians, Vol. 62, No. 1, 2012, pp. 10-29. http://dx.doi.org/10.3322/caac.20138

[2] G. G. Chabot, “Clinical Pharmacokinetics of Irinotecan,” Clinical Pharmacokinetics, Vol. 33, No. 4, 1997, pp. 245259.

http://dx.doi.org/10.2165/00003088-199733040-00001

[3] J.-F. Cote, S. Kirzin, A. Kramar, J.-F. Mosnier, M.-D. Diebold, I. Soubeyran, A.-S. Thirouard, J. Selves, P. Laurent-Puig and M. Ychou, "UGT1A1 Polymorphism Can Predict Hematologic Toxicity,” Clinical Cancer Research, Vol. 13, 2007, p. 3269.

http://dx.doi.org/10.1158/1078-0432.CCR-06-2290

[4] L. Iyer, S. Das, L. Janisch, M. Wen, J. Ramı'rez, T. Karrison, G. F. Fleming, E. E. Vokes, R. L. Schilsky and M. J. Ratain, "UGT1A1 Genotype and Irinotecan Disposition \& Toxicity,” The Pharmacogenomics Journal, Vol. 2, 2002, pp. 43-47. http://dx.doi.org/10.1038/sj.tpj.6500072

[5] L. Iyer, D. Hall, S. Das, M. A. Mortell, J. Ramirez, S. Kim, et al., "Phenotypegenotype Correlation of in Vitro SN-38 (Active Metabolite of Irinotecan) and Bilirubin Glucuronidation in Human Liver Tissue with UGT1A1 Promoter Polymorphism," Clinical Pharmacology \& Therapeutics, Vol. 65, 1999, pp. 576-582. http://dx.doi.org/10.1016/S0009-9236(99)70078-0

[6] F. Innocenti, C. Grimsley, S. Das, et al., "Haplotype Structure of the UDP-Glucuronosyltransferase 1A1 Promoter in Different Ethnic Groups,” Pharmacogenetics, Vol. 12, No. 9, 2002, pp. 725-733.

http://dx.doi.org/10.1097/00008571-200212000-00006

[7] H. Minami, K. Sai, M. Saeki, Y. Saito, S. Ozawa, K. Suzuki, N. Kaniwa, J. Sawada, T. Hamaguchi, N. Yamamoto, K. Shirao, Y. Yamada, H. Ohmatsu, K. Kubota, T. Yoshida, A. Ohtsu and N. Saijo, "Irinotecan Pharmaco- 
kinetics/Pharmacodynamics and UGT1A Genetic Polymorphisms in Japanese: Roles of UGT1A1*6 and *28," Pharmacogenet Genomics, Vol. 17, No. 7, 2007, pp. 497504. http://dx.doi.org/10.1097/FPC.0b013e328014341f

[8] E. A. Eisenhauer, P. Therasse, B. Bogaerts, L. H. Schwartz, D. Sargent, R. Ford, et al., "New Response Evaluation Criteria in Solid Tumors: Revised RECIST Guideline (Version 1.1)," European Journal of Cancer, Vol. 45, No. 2, 2009, pp. 228-247.

http://dx.doi.org/10.1016/j.ejca.2008.10.026

[9] "Common Terminology Criteria for Adverse Events," Version 4.0, DCTD, NCI, NIH, DHHS, 2009.

[10] L. Iyer1, S. Das, L. Janisch, M. Wen, J. Ramı'rez, T. Karrison, G. F. Fleming, E. E. Vokes, R. L. Schilsky and M. J. Ratain, "UGT1A1*28 Polymorphism as a Determinant of Irinotecan Disposition and Toxicity,” The Pharmacogenomics Journal, Vol. 2, No. 1, 2002, pp. 43-47.

[11] Y. Akiyama, et al., "Genetic Testing for UGT1A1*28 and *6 in Japanese Patients Who Receive Irinotecan Chemotherapy," Annals of Oncology, Vol. 19, No. 12, 2008, pp. 2089-2090. http://dx.doi.org/10.1093/annonc/mdn645

[12] X. Q. Zhang, G. K. Ao, Y. W. Wang, W. Yan, M. Wang, E. F. Chen, F. F. Yang and J. Yang, "Genetic Variants and Haplotypes of the UGT1A9, 1A7 and 1A1 Genes in Chinese Han,” Genetics and Molecular Biology, Vol. 35, No. 2, 2012, pp. 428-434.

http://dx.doi.org/10.1590/S1415-47572012005000036

[13] A. Zhang, Q. Xing, S. Qin, J. Du, L. Wang, L. Yu, X. Li, L. Xu, M. Xu, G. Feng, et al., "Intra-Ethnic Differences in Genetic Variants of the UGT-Glucuronosyltransferase 1A1 Gene in Chinese Populations," Pharmacogenomics Journal, Vol. 7, No. 5, 2007, pp. 333-338. http://dx.doi.org/10.1038/sj.tpj.6500424

[14] K. S. Tang, H. F. Chiu, H. H. Chen, H. L. Eng, C. J. Tsai, H. C. Teng and C. S. Huang, "Link between Colorectal Cancer and Polymorphisms in the Uridine-Diphosphoglucuronosyltransferase 1A7 and 1A1 Genes," World Journal of Gastroenterology, Vol. 11, 2005, pp. 32503254.

[15] Y. Y. Zhou, L. Y. Lee, S. Y. Ng, C. P. P. Hia, K. T. Low, Y. S. Chong and D. L. M. Goh, "UGT1A1 Haplotype Mutation among Asians in Singapore,” Neonatology, Vol. 96, 2009, pp. 150-155. http://dx.doi.org/10.1159/000209851

[16] M. Saeki, Y. Saito, H. Jinno, K. Sai, S. Ozawa, K. Kurose, N. Kaniwa, K. Komamura, T. Kotake, H. Morishita, et al., "Haplotype Structures of the UGT1A Gene Complex in a Japanese Population," Pharmacogenomics Journal, Vol. 6, 2006, pp. 63-75. http://dx.doi.org/10.1038/sj.tpj.6500335

[17] S. S. Yea, S. S. Lee, W. Y. Kim, K. H. Liu, H. Kim, J. H. Shon, I. J. Cha and J. G. Shin, "Genetic Variations and Haplotypes of UDP-Glucuronosyltransferase 1A Locus in a Korean Population,” Therapeutic Drug Monitoring, Vol. 30, No. 1, 2008, pp. 23-34.
http://dx.doi.org/10.1097/FTD.0b013e3181633824

[18] S. S. Thomas, S. S. Li, J. W. Lampe, J. D. Potter and J. Bigler, "Genetic Variability, Haplotypes, and htSNPs for Exons 1 at the Human UGT1A Locus," Human Mutation, Vol. 27, No. 7, 2006, p. e717. http://dx.doi.org/10.1002/humu.9432

[19] C. Schulz, V. Heinemann, A. Schalhorn, N. Moosmann, T. Zwingers, S. Boeck, C. Giessen and H.-J. Stemmler, "UGT1A1 Gene Polymorphism: Impact on Toxicity and Efficacy of Irinotecan-Based Regimens in Metastatic Colorectal Cancer," World Journal of Gastroenterology, Vol. 15, No. 40, 2009, pp. 5058-5066. http://dx.doi.org/10.3748/wjg.15.5058

[20] C.-Y. Liu, P.-M. Chen, T.-J. Chiou, J.-H. Liu, J. K. Lin, T.-C. Lin, W.-S. Chen, J.-K. Jiang, H.-S. Wang and W.-S. Wang, "UGT1A1*28 Polymorphism Predicts IrinotecanInduced Severe Toxicities without Affecting Treatment Outcome and Survival in Patients with Metastatic Colorectal Carcinoma,” Cancer, Vol. 112, No. 9, 2008, pp. 1932-1940. http://dx.doi.org/10.1002/cncr.23370

[21] K. K. Hahn, J. J. Wolff and J. M. Kolesar, "Pharmacogenetics and Irinotecan Therapy," American Journal of Health-System Pharmacy, Vol. 63, No. 22, 2006, pp. 2211-2217. http://dx.doi.org/10.2146/ajhp060155

[22] F. Innocenti, S. D. Undevia, L. Iyer, P. X. Chen, S. Das, M. Kocherginsky, T. Karrison, L. Janisch, J. Ramírez, C. M. Rudin, E. E. Vokes and M. J. Ratain, "Genetic Variants in the UDP-Glucuronosyltransferase 1A1 Gene Predict the Risk of Severe Neutropenia of Irinotecan,” Journal of Clinical Oncology, Vol. 22, No. 8, 2004, pp. 13821388. http://dx.doi.org/10.1200/JCO.2004.07.173

[23] E. Rouits, M. Boisdron-Celle, A. Dumont, O. Guérin, A. Morel and E. Gamelin, "Relevance of Different UGT1A1 Polymorphisms in Irinotecan-Induced Toxicity: A Molecular and Clinical Study of 75 Patients," Clinical Cancer Research, Vol. 10, No. 15, 2004, pp. 5151-5159.

http://dx.doi.org/10.1158/1078-0432.CCR-03-0548

[24] G. E. Palomaki, L. A. Bradley, M. P. Douglas, K. Kolor and W. D. Dotson, "Can UGT1A1 Genotyping Reduce Morbidity and Mortality in Patients with Metastatic Colorectal Cancer Treated with Irinotecan? An EvidenceBased Review," Genetics in Medicine, Vol. 11, No. 1, 2009, pp. 21-34. http://dx.doi.org/10.1097/GIM.0b013e31818efd77

[25] E. Marcuello, A. Altes, A. Menoyo, E. Del Rio, M. Gómez-Pardo and M. Baiget, "UGT1A1 Gene Variations and Irinotecan Treatment in Patients with Metastatic Colorectal Cancer,” British Journal of Cancer, Vol. 91, No. 4, 2004, pp. 678-682.

[26] G. Toffoli, E. Cecchin, G. Corona, et al., "The Role of UGT1A1*28 Polymorphism in the Pharmacodynamics and Pharmacokinetics of Irinotecan in Patients with Metastatic Colorectal Cancer,” Journal of Clinical Oncology, Vol. 24, No. 19, 2006, pp. 3061-3068. http://dx.doi.org/10.1200/JCO.2005.05.5400 